\title{
Diriving microalgal production in raceway systems to near optimal productivities
}

\author{
Rafael Muñoz-Tamayo ${ }^{1}$, Francis Mairet ${ }^{1,2}$, Olivier Bernard ${ }^{1,3}$
}

\begin{abstract}
In this paper, we propose a simple operational criterion for raceway systems that when integrated in a strategy of closed-loop control allows to attain microalgal productivities very near to the maximal producitivities. The strategy developed was tested numerically by using a mathematical model of microalgae growth in raceways. The model takes into account the dynamics of environmental variables such temperature and light intensity and their influence on microalgae growth.
\end{abstract}

\section{INTRODUCTION}

Microalgae are promising feedstocks for the production of high value compounds. The commercial use of microalgae includes applications in food industry and cosmetics. Moreover, microalgae have been identified as a renewable source for biodiesel production [1]. However, despite these favorable characteristics, microalgae production in large scale basis is probably carried out far from an optimal working mode. Here, we mainly refer to the technology of raceways, which are nowadays the systems generally used for large microalgae production.

The difficulty of achieving optimal productivities of microalgae in outdoor systems results from the high interaction of phenomena that take place during growth and the low level of control that we have on them. This factor makes the whole process inefficient under an environment that is fluctuating by nature. The challenge of optimizing microalgal culture systems is a broad endeavour that includes reactor design and strain selection. Furthermore, once the reactor configuration and the microalgal strain have been chosen, optimal performances can be achieved by acting on operational variables, such as nutrient feeding rate. In this aspect, mathematical models are powerful tools, because optimization can be model driven. Since microalgal metabolism is mainly influenced by nutrient availability, light intensity and temperature, several models have been developed to account for these factors [2], [3], [4]. A work of synthesis has been performed to provide a representative model microalgal dynamics by keeping a relative simple structure [6] that might be suitable for control processes.

The task of bringing a process close to optimality by acting on the inputs of the system is the realm of optimal control. The optimal control problem that we are considering consists in finding the time evolution of the manipulated variables maximizing a given criterion on a finite time horizon. This

1 BIOCORE-INRIA, BP93, 06902 Sophia-Antipolis Cedex, France rafael.munoz_tamayodinria.fr

2 Departamento de Matemática, Universidad Técnica Federico Santa María, Valparaíso, Chilefrancis.maireteusm.cl

${ }^{3}$ LOV-UPMC-CNRS, UMR 7093, Station Zoologique, B.P. 2806234 , Villefranche-sur-mer, France olivier.bernardeinria.fr problem can be solved by indirect methods such as the Pontryagins maximum principle or by direct methods (numerical optimization). The advantage of using Pontryagins maximum principle is that of providing an analytic solution. In this respect, a theoretical study on a simplified model of microalgae growth provided guidelines on the form of the controller to reach an optimal productivity [7]. A series of simplifications were needed to provide a very simple model suitable for applying the Pontryagins maximum principle. The distance between the proposed optimal strategy and the optimal strategy for a more realistic model is therefore difficult to assess.

For complex models, the application of the Pontryagins maximum principle is not straightforward. Hence, methods based on numerical optimization are, in practice, the most used. In the standard form, the numerical approach takes place in open loop fashion, without taking into account the real state of the system. For a real implementation, however, available measurements (either online or offline) must be used to compensate for disturbances and to correct model mismatches [8]. An example of this type of strategy is the predictive control approach, in which the optimal control problem is solved online. This strategy was developed for the optimization of biomass and oil productivities for the heterotrophic microalgae Auxenochlorella protothecoides [9]. This approach, however,is computationally expensive and requires sophisticated algorithms of adaptation and proof of process stability is lacking.

A practical alternative for optimizing system performance is to translate the optimization problem into a regulatory problem. The objective then consists in finding a variable that when regulated maintains the system close to optimality [10]. In the case of photobioreactors, the phenomenon of light transfer to the culture governs the performance of the system [11]. Based on this principle, we propose in this work a simple operational criterion which when regulated to an adequate set point maintains the performance of a raceway near to optimal operation. The proposed strategy has the advantage to be straightforward to implement in a classical closed loop control.

As a basis, we use the model proposed by [6] for a planar culturing device in combination to a model describing lipid production under nitrogen limitation [15], [16]. These models have shown to reproduce experimental data of lab scale systems. Here, we extend such models to account for characteristics of raceway systems. Our in silico case study takes the configuration of a pilot-scale open raceway (Algotron) located at INRA-LBE, France. 


\section{MODELING}

Under the assumption that nitrogen and light are the limiting factors for the growth of microalgae, we combined the biomass model from [6] to the lipid production model proposed in [15], [16]. It results in the following mass balance equations for a completely mixed reactor at constant volume $V$

$$
\begin{aligned}
\dot{s} & =f_{i} s_{i n} / V-f_{i} s / V-\rho(\cdot) x, \\
\dot{q}_{n} & =\rho(\cdot)-(\mu(\cdot)-R(\cdot)) q_{n}, \\
\dot{x} & =\left(\mu(\cdot)-f_{i} / V-R(\cdot)\right) x, \\
\dot{x_{l}} & =\beta q_{n} \mu(\cdot) x-\gamma \rho(\cdot) x-r_{0} \phi_{T} x_{l}-f_{i} x_{l} / V,
\end{aligned}
$$

where $s\left(\mathrm{~g} \mathrm{~N} \mathrm{~m}^{-3}\right)$ is the extracellular nitrogen concentration and $q_{n}\left(\mathrm{~g} \mathrm{~N}\left(\mathrm{~g} \mathrm{C}^{-1}\right)\right.$ is the internal nitrogen quota. The model includes the concentration of the total carbon biomass $x\left(\mathrm{~g} \mathrm{C} \mathrm{m}^{-3}\right)$ and the carbon biomass concentration of storage lipids $x_{l}\left(\mathrm{~g} \mathrm{C} \mathrm{m}^{-3}\right)$. The influent nitrogen concentration is $s_{\text {in }}\left(\mathrm{g} \mathrm{N} \mathrm{m}^{-3}\right)$. The influent flow rate is $\left(\mathrm{m}^{3} \mathrm{~d}^{-1}\right)$. The functions $\mu(\cdot)$ and $\rho(\cdot)$ represent the kinetics of growth rate and nitrogen uptake.

The temperature exerts a strong influence on the behaviour of microalgae systems, in particular in outdoor raceways. This effect is included in the model in two manners. Firstly, it is assumed, in line with [3], that temperature has an homogeneous effect on uptake, growth and respiration rates. Secondly, following the work of [17], the chlorophyll:nitrogen ration (Chl a:N) ratio was set to be dependent on the temperature and light. The equations are detailed later on.

To model the growth rate, the following is assumed: $(i)$

1) Microalgal growth is uncoupled dynamically to nutrient uptake. Growth kinetics follows the cell quota model of Droop [18].

2) Light intensity is distributed spatially in the raceway. The absorption of light in the raceway follows the Lambert-Beer law. Thus, for a given depth $z$, the corresponding light intensity $I_{z}\left(\mu \mathrm{E} \mathrm{m}^{-2} \mathrm{~s}^{-1}\right)$ satisfies

$$
I_{z}=I_{0} \exp \left(-\xi_{z}\right),
$$

where $I_{0}\left(\mu \mathrm{E} \mathrm{m}^{-2} \mathrm{~s}^{-1}\right)$ is the incident light and $\xi$ is the light attenuation factor, which depends on the chlorophyll concentration $\mathrm{Chl}$

$$
\xi=a \mathrm{Chl}+b .
$$

At the bottom of the reactor $z=L$. The term $\xi L$ is known as optical depth $(\lambda)$. It should be noted that $I_{0}$ varies in time in an oscillatory fashion. Its amplitude depends on the season and the geographical location. For a given day, $I_{0}$ follows an increasing behaviour until noon, then decreases until midnight.

3) Light intensity affects the growth rate. This effect is described by a Monod type kinetics. For a given depth $z(0 \leq z \leq L)$ with intensity $I_{z}$, the growth rate at hypothetical infinite nitrogen quota is

$$
\mu_{z}=\tilde{\mu} \frac{I_{z}}{I_{z}+K_{s I}} .
$$

Finally, the growth rate is represented by an average growth rate obtained by integration of (7) along the raceway depth. The resulting equation for the growth rate reads

$$
\mu(\cdot)=\overline{\bar{\mu}} \phi_{T}\left(1-\frac{Q_{0}}{q_{n}}\right),
$$

with

$$
\begin{gathered}
\overline{\bar{\mu}}=\frac{\tilde{\mu}}{\xi L} \ln \frac{I_{0}+K_{S I}}{I_{0} e^{-\xi L}+K_{s I}}, \\
\phi_{T}=\frac{\left(T-T_{\max }\right)\left(T-T_{\min }\right)^{2}}{\left(T_{\mathrm{opt}}-T_{\min }\right)\left[\left(T_{\mathrm{opt}}-T_{\min }\right)\left(T-T_{\mathrm{opt}}\right)-\left(T_{\mathrm{opt}}-T_{\mathrm{max}}\right)\left(T_{\mathrm{opt}}+T_{\min }-2 T\right)\right]} .
\end{gathered}
$$

The term $\phi_{T}$ represents the effect of the temperature $(T(\mathrm{C}))$. It is described by the model developed for bacteria by [19] and validated for microalgae by [5].

Nitrogen uptake rate $(\rho(\cdot))$ is modeled by a modified Michaelis-Menten kinetics [15].

$$
\rho(\cdot)=\bar{\rho} \phi_{T} \frac{s}{s+K_{s}}\left(v+(1-v) \frac{\bar{I}^{m}}{\bar{I}^{m}+\varepsilon_{I}^{m}}\right)\left(1-\frac{q_{n}}{Q_{l}}\right) .
$$

The nitrogen uptake rate is expressed as a function of the average irradiance in the raceway $\bar{I}$. Here, it is considered that nutrient uptake is regulated by the internal nitrogen quota, i.e., when the cells are nutrient saturated, uptake rate stops. Additionally, the equation includes a light regulating factor (in the form of a Hill-type function). Therefore, when the cells enter to the dark period, the nutrient uptake rate exhibits a slowdown.

The model includes an overall respiration rate $R$, that gathers maintenance respiration and biosynthesis cost (assumed to be proportional to nitrogen uptake rate):

$$
R(\cdot)=r_{0} \phi_{T}+\varphi \rho(\cdot)
$$

where $r_{0}$ is the maintenance respiration and $\varphi$ is a biosynthesis cost coefficient.

Additionally, it is assumed that chlorophyll concentration (Chl) is correlated to particulate nitrogen $\left(x q_{n}\right)$ [6]. The Chl:N ratio $\left(\theta_{N}\right)$ is influenced by light and temperature following [17]

$$
\theta_{N}^{-1}=\left(g_{1}-g_{2} T\right)+g_{3} \bar{I} \exp \left(-g_{4} T\right) .
$$

In this equation, it is implicitly assumed that the cells are photoacclimated at the average light intensity $\bar{I}$.

Environmental variables, notably light intensity (solar irradiance) and temperature govern reactor performance. These two variables incorporated in the kinetics of growth and nitrogen uptake can be accessible from online sensors or meteorological stations. In addition, mathematical models have been developed to predict light intensity [20] and raceway temperature [21] for a given location. In the present study, mathematical modeling supported by meteorological data was used for the location of Narbonne, France. The results presented here correspond to typical environmental conditions for the month of June.

Model parameters were taken from studies on the microalgae Isochrysis aff. galbana, when available. The parameters describing $\phi_{T}$ are those obtained for Nannochloropsis oceanica [5]. The values of model parameters are given in Table I. 
TABLE I

VALUES OF MODEL PARAMETERS

\begin{tabular}{|c|c|c|}
\hline \multicolumn{2}{|c|}{ Parameters } & Value \\
\hline$\alpha$ & Protein synthesis coefficient & $3.0 \mathrm{~g} \mathrm{C}\left(\mathrm{g} \mathrm{N}^{-1}\right.$ \\
\hline$\beta$ & Fatty acid synthesis coefficient & $3.80 \mathrm{~g} \mathrm{C}\left(\mathrm{g} \mathrm{N}^{-1}\right.$ \\
\hline$\varepsilon_{I}$ & Dissociation light constant & $50 \mu \mathrm{E} \mathrm{m}^{-2} \mathrm{~s}^{-1}$ \\
\hline$\varphi$ & Biosynthesis cost coefficient & $1.30 \mathrm{~g} \mathrm{C}\left(\mathrm{g} \mathrm{N}^{-1}\right.$ \\
\hline$\gamma$ & $\begin{array}{l}\text { Fatty acid mobilization } \\
\text { coefficient }\end{array}$ & $2.90 \mathrm{~g} \mathrm{C}(\mathrm{g} \mathrm{N})^{-1}$ \\
\hline$v$ & $\begin{array}{l}\text { Reduction factor of nitrogen } \\
\text { uptake during night }\end{array}$ & 0.19 \\
\hline$\tilde{\mu}$ & $\begin{array}{l}\text { Theoretical maximum } \\
\text { specific growth rate }\end{array}$ & $2.11 \mathrm{~d}^{-1}$ \\
\hline $\bar{\rho}$ & Maximum uptake rate & $0.10 \mathrm{~g} \mathrm{~N}(\mathrm{~g} \mathrm{C} \mathrm{d})^{-1}$ \\
\hline$a$ & $\begin{array}{l}\text { Light attenuation due } \\
\text { to chlorophyll }\end{array}$ & $2.0 \mathrm{~m}^{2}\left(\mathrm{~g} \mathrm{Chl}^{-1}\right.$ \\
\hline$b$ & $\begin{array}{l}\text { Light attenuation due to } \\
\text { background turbidity }\end{array}$ & $0.087 \mathrm{~m}^{-1}$ \\
\hline$g_{1}$ & Coefficient Eq. (11) & $16.74 \mathrm{~g} \mathrm{~N}\left(\mathrm{~g} \mathrm{Chl}^{-1}\right.$ \\
\hline$g_{2}$ & Coefficient Eq. (11) & $0.39 \mathrm{~g} \mathrm{~N}\left(\mathrm{~g} \mathrm{Chl} \mathrm{C}^{-1}\right.$ \\
\hline$g_{3}$ & Coefficient Eq. (11) & $\begin{array}{c}0.0014 \\
\mathrm{~g} \mathrm{~N}\left(\mathrm{~g} \mathrm{Chl} \mu \mathrm{E} \mathrm{m}^{-2} \mathrm{~s}^{-1}\right)^{-1}\end{array}$ \\
\hline$g_{4}$ & Coefficient Eq. (11) & $0.0015(\mathrm{C})^{-1}$ \\
\hline$K_{s}$ & Nitrogen saturation constant & $0.018 \mathrm{~g} \mathrm{~N} \mathrm{~m}^{-3}$ \\
\hline$K_{s I}$ & Light saturation constant & $150 \mu \mathrm{E} \mathrm{m}^{-2} \mathrm{~s}^{-1}$ \\
\hline$L$ & Pond depth. & $0.30 \mathrm{~m}$ \\
\hline$m$ & Hill coefficient & 3.0 \\
\hline$Q_{l}$ & Saturation cell quota & $0.25 \mathrm{~g} \mathrm{~N}\left(\mathrm{~g} \mathrm{C}^{-1}\right.$ \\
\hline$Q_{0}$ & Minimal nitrogen cell quota & $0.05 \mathrm{~g} \mathrm{~N}\left(\mathrm{~g} \mathrm{C}^{-1}\right.$ \\
\hline$r_{0}$ & Maintenance respiration rate & $0.01 \mathrm{~d}^{-1}$ \\
\hline$s_{\text {in }}$ & Influent nitrogen concentration & $50 \mathrm{~g} \mathrm{~N} \mathrm{~m}^{-3}$ \\
\hline$S$ & Pond surface & $57 \mathrm{~m}^{2}$ \\
\hline$T_{\min }$ & $\begin{array}{l}\text { Lower temperature for } \\
\text { microalgae growth }\end{array}$ & $-0.20 \mathrm{C}$ \\
\hline$T_{\max }$ & $\begin{array}{l}\text { Upper temperature for } \\
\text { microalgae growth }\end{array}$ & $33.30 \mathrm{C}$ \\
\hline$T_{\mathrm{opt}}$ & $\begin{array}{l}\text { Temperature at which } \\
\text { growth rate is maximal }\end{array}$ & $26.70 \mathrm{C}$ \\
\hline$V$ & Raceway volume & $17.10 \mathrm{~m}^{3}$ \\
\hline
\end{tabular}

\section{DRIVING RACEWAY OPERATION TO NEAR OPTIMAL PERFORMANCE.}

\section{A. Optimal problem statement}

In this study, we are interested in designing a control law on the input flow rate $\left(f_{i}\right)$ that maximizes either the biomass productivity $\left(\mathrm{P}_{x}\right)$ or the lipid productivity $\left(\mathrm{P}_{l}\right)$ for a given time horizon $t_{f}$. The maximal productivities can be obtained by solving an optimal control problem that can be formulated as follows

$$
\begin{aligned}
\max _{f_{i}(t)} & \int_{t_{0}}^{t_{f}} \psi\left(t, \mathbf{x}(t), f_{i}(t)\right) d t . \\
\text { s.t. } & \\
& 0 \leq f_{i}(t) \leq f_{\max } \\
\dot{\mathbf{x}} & =\mathbf{g}\left(\mathbf{x}, f_{i}, t\right), \mathbf{x}(0)=\mathbf{x}_{0} .
\end{aligned}
$$

With $\mathbf{x}$ the state vector and $f_{\max }$ the upper bound of the input flow rate. If the purpose of the controller is to optimize biomass productivity $\mathrm{P}_{x}$, then

$$
\psi\left(t, \mathbf{x}(t), f_{i}(t)\right)=f_{i}(t) x(t) .
$$

If the objective is to optimize lipid productivity $\mathrm{P}_{l}$, then

$$
\psi\left(t, \mathbf{x}(t), f_{i}(t)\right)=f_{i}(t) x_{l}(t) .
$$

For the sake of clarity, we will call $\mathrm{CP}_{x}$ the optimal controller that maximizes (13) and $\mathrm{CP}_{l}$ the optimal controller that maximizes (14). The Matlab toolbox DOTcvpSB [22] was used for solving (13),(14) numerically. DOTcvpSB uses the approach of sequential discretization (control vector parametrization) to solve the non-linear programming (NLP) problem.

\section{B. Quasi optimal closed loop control}

Solving the optimal control problems (13),(14) might be computationally expensive and difficult to implement in practice. For a real implementation, it will be desirable to identify a controlled variable that when regulated towards a set point will ensure that the system operates close to optimality. In this respect and since light transfer is a crucial phenomenon of the process of microalgal growth, we propose the efficiency of light absorption $\left(\eta_{L}\right)$ to be such a controlled variable.

$$
\eta_{L}=\frac{I_{0}-I_{L}}{I_{0}}=1-\exp (-\xi L)
$$

As it will be shown hereafter, this simple controller has a very good ability to maintain the system close to the optimal solution. In fact, several strategies where tested based on preliminary studies, and $\eta_{L}$ turned out to present the best trade-off between simplicity and efficiency.

For a given microalgae, there exists a set point $\eta_{L}^{*}$ that maintains the system near to optimal productivities. In this study a set point $\eta_{L}^{*}=0.95$ was selected. Note that regulating $\eta_{L}$ implies the regulation of the optical depth $\lambda$. Given the form of the attenuation factor (6), regulating the optical depth is equivalent to regulating the Chlorophyll concentration. For $\eta_{L}^{*}=0.95$, the set point for Chlorophyll concentration is $\mathrm{Chl}^{*}=4.95 \mathrm{~g} \mathrm{Chl} \mathrm{m}^{-3}$. This result is very convenient because during darkness we can still regulate the Chlorophyll concentration to $\mathrm{Chl}^{*}$ in such a way that when $I_{0}>0$ the efficiency of light absorption will be close to $\eta_{L}^{*}$.

In the following, we show by means of numerical simulation, the performance of the raceway by regulating $\eta_{L}$ to the set point $\eta_{L}^{*}$. This regulation can be achieved by any adequate feedback controller. In this work, we use a standard PI controller. Since our premise is that this controller brings the system to work almost optimally, we call it a quasi optimal (QO) controller.

\section{RESULTS}

\section{A. Comparison of the $Q O$ controller to optimal strategy}

Figure 1 displays the responses of the state variables and the lipid and biomass productivities $\left(\mathrm{P}_{l}, \mathrm{P}_{x}\right)$ when applying the QO controller and the optimal $\mathrm{CP}_{x}$ controller for a time period of 30 days. To calculate the productivities, it was assumed that carbon contributes to the $56 \%$ of ash-free dry weight [23]. The productivities are divided by the surface of the raceway and the time. The QO control controller brings 

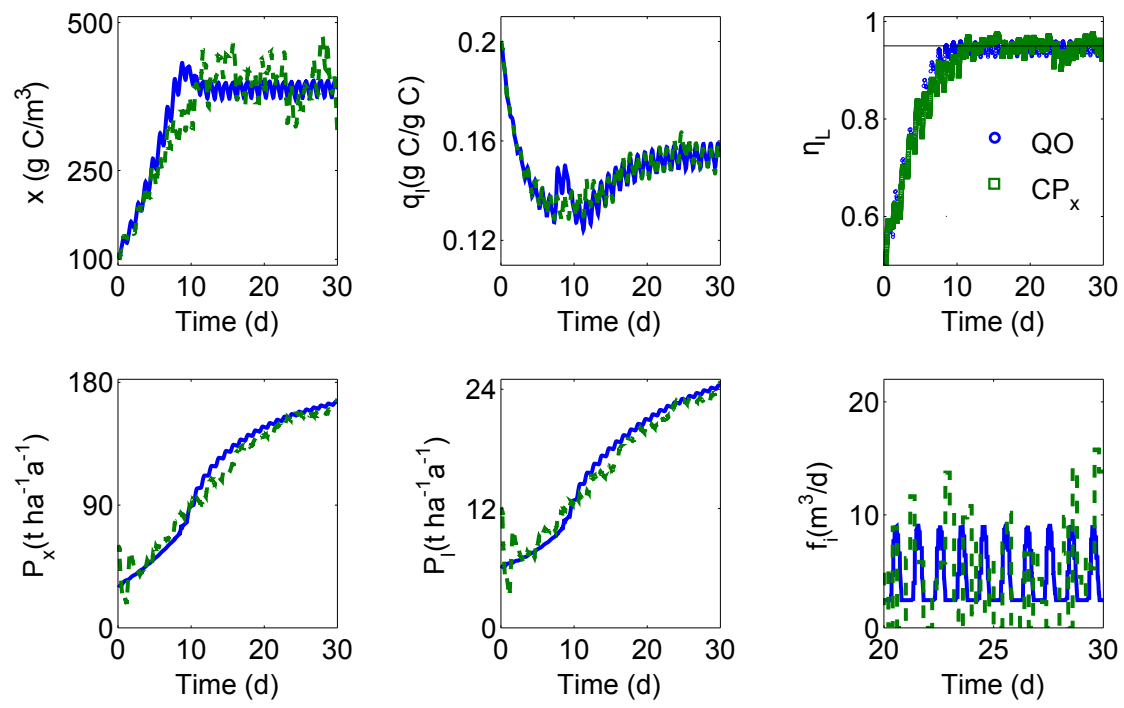

Fig. 1. Trajectories of state variables and productivities given by the $\mathrm{QO}$ controller (solid blue line) and by the optimal controller $\mathrm{CP}_{x}$ (dashed green line). The productivities are given in dry weight basis. The biomass and lipid productivities given by the QO controller are both $98 \%$ of those provided by $\mathrm{CP}_{x}$. The top right plot shows the evolution $\eta_{L}$ during the light period. The horizontal continuous line corresponds to the set point $\eta_{L}^{*}=0.95$.

the efficiency of light absorption very close to the defined set point. At $t=6.5 \mathrm{~d}, \eta_{L}$ is $95 \%$ of $\eta_{L}^{*}$. The maximal biomass productivity obtained with the optimal controller $\mathrm{CP}_{x}$ is 168 tons dry weight $\mathrm{ha}^{-1} \mathrm{a}^{-1}$. This value is consistent with productivities reported in the literature [1], [23]. Importantly, the biomass and lipid productivity provided by the QO controller were both $98 \%$ of those given by the controller $\mathrm{CP}_{x}$ (Table II). After $25 \mathrm{~d}$, the final lipid quota $\left(q_{l}=x_{l} / x\right)$ oscillates with a maximal value of $16 \%$. This relative low level of lipids is due to the fact that many of the parameters used in the model were taken from studies with I. galbana, which is known to have a low lipid content.

To have an assessment of the maximal lipid productivity that can be attained, the optimal controller $\mathrm{CP}_{l}$ that solves (14) was calculated. For the model parameters used in our case study, it resulted that optimizing lipid productivity was equivalent to optimizing biomass productivity. Thus, the response of the system behaviour when applying the $\mathrm{CP}_{l}$ controller was very similar to the response obtained when applying the $\mathrm{CP}_{x}$ controller. This result is interesting because it is often claimed the conflict between optimizing lipid productivity and optimizing biomass productivity. Indeed, such a conflict occurs when light is constant. For a diurnal light cycle, however, our results suggest that there is not discrepancy between optimizing lipid productivity and optimizing biomass productivity. For both performance indexes, the cells should growth as much as they can in the light period to accumulate enough carbon. The higher the biomass the concentration, the higher the available carbon source that can be potentially directed to the lipid pool.

The results presented here are very promising. We show that the QO controller performs as well as the optimal controllers, confirming our hypothesis that controlling the efficiency of light absorption $\left(\eta_{L}\right)$ makes it possible to attain high productivities both in lipid and biomass. The response of the QO controller suggests that an optimal strategy consists in driving the biomass concentration to a certain value and to allow it oscillate around this point. This result is consistent with the work developed by [12] and the theoretical results presented by [7], where an optimal controller was developed by forcing the biomass concentration to fulfill a periodicity condition.

\section{B. Is the strict compensation condition relevant for diurnal light cycles?}

In closed photobioreactors under light constant regime, it has been proposed that maximal productivities are attained when the light intensity at the rear of the reactor equals the light of compensation $\left(\mathrm{G}_{c}\right)$, defined as the minimum value of light intensity required to guarantee a positive net growth rate [13]. This condition is called the strict compensation condition. The light intensity at which the compensation occurs is often expressed as a constant parameter. This may be the case when the incident light intensity is constant. However, we might notice that for a varying light system, the light of compensation depends on the actual state of the system and thus there is not a fixed value that will bring the system to operate under the strict compensation condition.

For outdoor raceways, where microalgae are exposed to long periods of darkness, respiration affects negatively growth. It is clear that in the dark period, the compensation condition do not play any role on the reactor performance. When the incident light is higher than zero, the strict compensation condition is such that $\mu_{L}=R$. The light of compensation is thus a dynamic operational variable that depends of the state of the system. The optical depth of the reactor must then be adjusted accordingly to reach the light of compensation at the rear of the reactor. Note that if the reactor volume is constant, the regulation acts on the attenuation factor $\xi$. This strategy, however, may suffer 
of reachability problems, as experienced in the study of [14], where the light at the bottom of the photobioreactor could not be maintained at the defined set point due to the dynamic boundary imposed by the growth rate. To enlarge the discussion in this point, we assess by means of simulations if the strict compensation condition could be fulfilled in a diurnal light cycle and if it is relevant to attain such a condition to achieve maximal productivities. The following optimal control problem was defined

$$
\min _{f_{i}(t)} \int_{t_{0}}^{t_{f}}\left(\mu_{L}-R\right)^{2} d t .
$$

The controller optimizing (16) is called $\mathrm{CP}_{c}$.

Figure 2 shows the ratio between the growth rate at the rear of the raceway $\left(\mu_{L}\right)$ and the respiration rate $(\mathrm{R})$. The results are given for the optimal controller $\mathrm{CP}_{c}$. It is observed that, for the light period, the growth rate at the rear of the raceway is higher than the respiration rate and that the compensation condition is not strictly fulfilled all the time. The results indicate that attaining the strict compensation throughout the day might be no physically possible due to the dynamic bound imposed by the growth rate.

The optimal controller $\mathrm{CP}_{c}$ resulted in biomass and lipid productivities that were, $100 \%$ of those obtained with the optimal controllers $\mathrm{CP}_{x}$ and $\mathrm{CP}_{l}$. Our results suggest that the closest the system is to the compensation condition the closest the system operates optimally. However, the results also indicates that for a photobioreactor subject to the diurnal light cycle, the strict compensation condition is not a necessary condition to be fulfilled for achieving maximal productivities. We have also verified that trying to impose a compensation condition valid around the midday light peak could be inefficient resulting in low productivities.

We must note that when the respiration rate is negligible, the strict compensation condition became $\mu_{L} \approx 0$ implying that $I_{L} \approx 0$. Here, the compensation condition implies almost full absorption of light which is rather difficult to maintain throughout the day. Due to the limitation of reachability of the strict compensation condition and the difficulty associated to the online determination of $\mu_{L}$ and $\mathrm{R}$, we suggest that the strict compensation condition is not a practical criterion for the design of control strategies. By contrary, the strategy that we proposed of controlling the efficiency of light absorption $\eta_{L}$ is technically feasible for real implementation and provides almost optimal productivities. In the near future, an optimal framework of harvesting strategies will be proposed complementary to the QO control.

\section{Comparison of open loop configurations to optimal strat- egy}

We were interested to assess the performance of the raceway in open loop (OL) configuration. To this end, the model was simulated initially with an input flow rate $f_{i}=5.13 \mathrm{~m}^{3}$ $\mathrm{d}^{-1}$ (dilution rate $\mathrm{D}=0.30 \mathrm{~d}^{-1}$ ), which is a typical value [24]. The lipid and biomass productivities were, respectively, $54 \%$ and $59 \%$ of those obtained with the optimal controllers

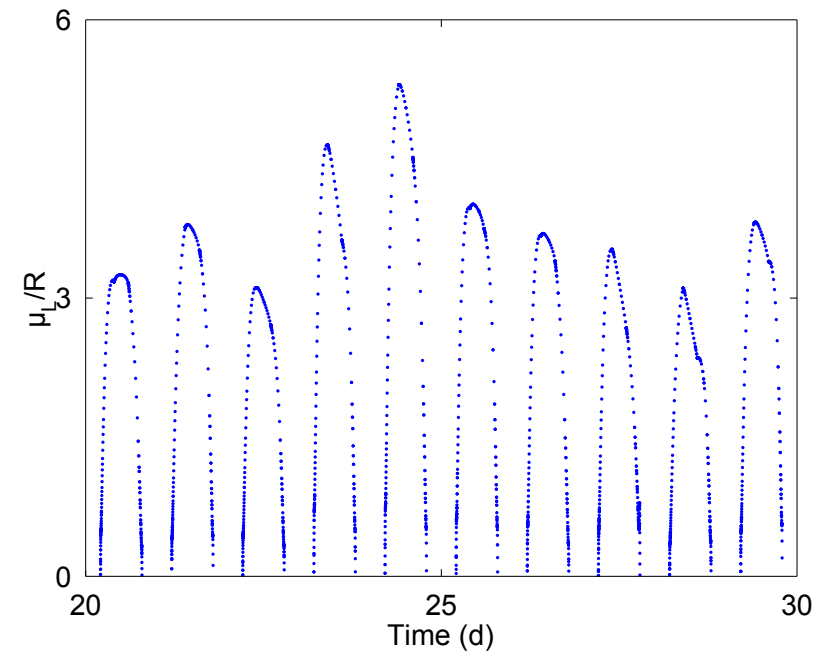

Fig. 2. The strict compensation condition $\left(\mu_{L} / \mathrm{R}=1\right)$ is not attained along the day. Response obtained for the optimal controller $\mathrm{CP}_{c}$.

$\mathrm{CP}_{x}$ and $\mathrm{CP}_{l}$. This means that for an open loop configuration, a wrong choice of the input flow rate will imply an unsatisfactory performance. To perform a fair comparison, in addition to the optimal controllers previously calculated, the optimal control problem (13) was solved by setting a constant flow rate. The optimal flow rate was found to be $3.22 \mathrm{~m}^{3} \mathrm{~d}^{-1}$ $\left(\mathrm{D}=0.19 \mathrm{~d}^{-1}\right)$ and the resulting biomass productivity was $93 \%$ of the one obtained with the optimal controller $\mathrm{CP}_{x}$. Table II summarizes the comparison of the performances of different controllers and configurations evaluated (including the QO controller) relative to the optimal productivities.

The relative high productivity obtained with the optimal constant flow rate is not surprising, since experimental studies on artificial photobioreactors [14] have shown that with an adequate constant flow rate it is possible to attain high productivities. This result may suggest that, when the microalgae are not nutrient limited, the environmental conditions, namely light intensity and temperature exert such a strong influence on the system behaviour that the improvement of the performance that can be reached by manipulating the dilution rate is only marginal. This finding might, at first sight, discourages the endeavour of developing any control strategy for raceways systems, since it appears that even with a constant flow rate, a satisfactory performance can be attained. This result, however, must be taken with caution. Indeed, we argue in favor of the QO controller over the other control strategies analyzed. The QO controller has the advantage to operate in closed loop fashion. Hence, it can be easily tuned for a real scenario that is subjected to disturbances and technical failures. The optimal controllers can also be in closed loop fashion. However, its implementation is more demanding than that of the QO controller. If the optimal controllers are used in open loop, they are not adapted to account for model uncertainty and potentian disturbances, which can lead to suboptimal operation. Figure 3 displays the productivities given by the 
QO controller and the optimal controller $\mathrm{CP}_{x}$ considering an uncertainty in the model parameters. The value of $\tilde{\mu}$ was decreased $30 \%$ of the value used originally to calculate the optimal controller. It is observed that the QO controller provided a biomass productivity that is $17 \%$ higher than that provided by the controller $\mathrm{CP}_{x}$. It should be noted that this result was achieved with a simple PI controller. We expect that by using a nonlinear controller based on the ligth efficiency, the productivity might be even better. The design of such a nonlinear controller is one of the perspectives of this work.

\section{TABLE II}

PRODUCTIVITY PERFORMANCE OF OPEN LOOP (OL) CONFIGURATION AND CLOSED LOOP CONTROLLER. THE RESULTING PERFORMANCE IS PRESENTED RELATIVE TO THE OPTIMAL STRATEGY.

\begin{tabular}{|c|c|c|}
\hline & $100 \mathrm{P}_{x} / \mathrm{P}_{x}^{*}$ & $100 \mathrm{P}_{l} / \mathrm{P}_{l}^{*}$ \\
\hline $\mathrm{QO}$ & $98 \%$ & $98 \%$ \\
\hline $\mathrm{CP}_{c}$ & $100 \%$ & $100 \%$ \\
\hline OL: $f_{i}^{\star}=3.22 \mathrm{~m}^{3} \mathrm{~d}^{-1}$ & $93 \%$ & $91 \%$ \\
\hline $\mathrm{OL}: f_{i}=5.13 \mathrm{~m}^{3} \mathrm{~d}^{-1}$ & $54 \%$ & $59 \%$ \\
\hline
\end{tabular}

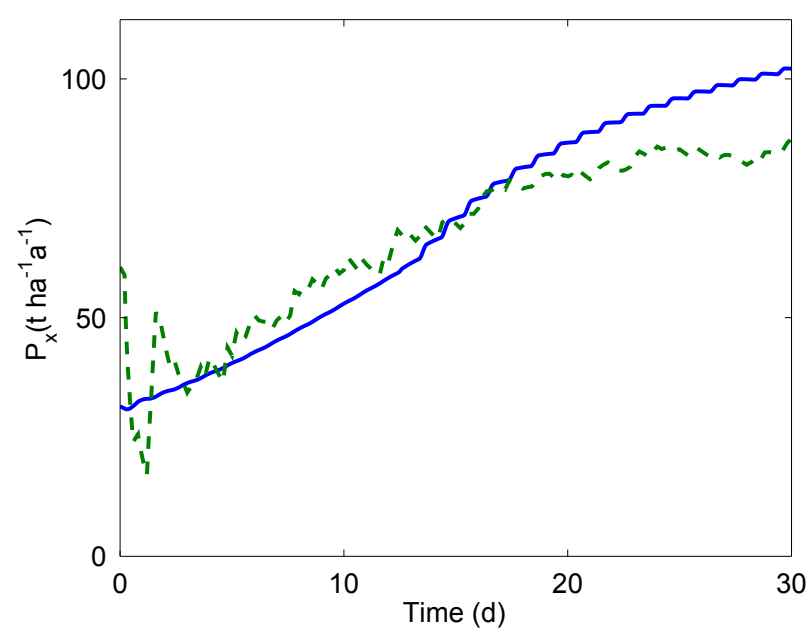

Fig. 3. Productivities provided by the QO controller (solid blue line) and the optimal controller $\mathrm{CP}_{x}$ (dashed green line) under parameter uncertainty. The value of $\tilde{\mu}$ was decreased $30 \%$.

\section{CONCLUSIONS}

Controlling the efficiency of light absorption makes it possible to attain maximal productivities. The overall performance of the QO controller developed here and its practical advantages for real implementation makes it a suitable control strategy for optimizing microalgae production in raceways.

\section{ACKNOWLEDGMENT}

We thank Bruno Sialve and Dr. Eric Latrille for providing us with relevant data of the Algotron raceway at LBE-INRA, and Dr. Doris Brockmann for kindly sharing her Matlab code of raceway modeling.

\section{REFERENCES}

[1] Y. Chisti, Biodiesel from microalgae., Biotechnol Adv 25 (3) (2007) 294-306.

[2] J. F. Cornet, C. G. Dussap, J. B. Gros, C. Binois, C. Lasseur, A simplified monodimensional approach for modeling coupling between radiant light transfer and growth-kinetics in photobioreactors, Chen Eng Sci 50 (1995) 1489-1500.

[3] R. J. Geider, H. L. MacIntyre, T. M. Kana, A dynamic regulatory model of phytoplanktonic acclimation to light, nutrients, and temperature, Limonol. Oceanogr. 43 (1998) 679-694.

[4] O. N. Ross, R. J. Geider, New cell-based model of photosynthesis and photo-acclimation: accumulation and mobilisation of energy reserves in phytoplankton, Mar Ecol Prog Ser. 383 (2009) 53-71.

[5] O. Bernard, B. Remond, Validation of simple model accounting for light and temperature effect on microalgal growth, Bioresour Technol 123 (2012) 520-257.

[6] O. Bernard, Hurdles and challenges for modelling and control of microalgae for $\mathrm{CO} 2$ mitigation and biofuel production, Journal of Process Control 21 (2011) 1378-1389.

[7] F. Grognard, A. Akhmetzhanov, P. Masci, O. Bernard, Optimization of a photobioreactor biomass production using natural light, in: Proc. 49th IEEE Conf. Decision and Control (CDC), 2010, pp. 4691-4696.

[8] B. Chachuat, B. Srinivasan, D. Bonvin, Adaptation strategies for realtime optimization, Computers and Chemical Engineering 33 (2009) $1557-1567$.

[9] H. De la Hoz Siegler, W. C. McCaffrey, R. E. Burrell, A. Ben-Zvi, Optimization of microalgal productivity using an adaptive, non-linear model based strategy., Bioresour Technol 104 (2012) 537-546.

[10] S. Skogestad, Plantwide control: the search for the self-optimizing control structure, Journal of Process Control 10 (2000) 487-507.

[11] A. Richmond, Principles for attaining maximal microalgal productivity in photobioreactors: an overview, Hydrobiologia 512 (2004) 33-37.

[12] J.-F. Cornet, Calculation of optimal design and ideal productivities of volumetrically lightened photobioreactors using the constructal approach, Chemical Engineering Science 65 (2010) 985-998.

[13] H. Takache, G. Christophe, J.-F. Cornet, J. Pruvost, Experimental and theoretical assessment of maximum productivities for the microalgae Chlamydomonas reinhardtii in two different geometries of photobioreactors., Biotechnol Prog 26 (2010) 431-440.

[14] M. Cuaresma, M. Janssen, E. J. van den End, C. Vlchez, R. H. Wijffels, Luminostat operation: a tool to maximize microalgae photosynthetic efficiency in photobioreactors during the daily light cycle?, Bioresour Technol 102 (2011) 7871-7878.

[15] F. Mairet, O. Bernard, T. Lacour, A. Sciandra, Modelling microalgae growth in nitrogen limited photobiorector for estimating biomass, carbohydrate and neutral lipid productivities, in: Proc. 18th World Congress The International Federation of Automatic Control, Milano, Italy, 2011.

[16] F. Mairet, O. Bernard, P. Masci, T. Lacour, A. Sciandra, Modelling neutral lipid production by the microalga Isochrysis aff. galbana under nitrogen limitation., Bioresour Technol 102 (2011) 142-149.

[17] R. J. Geider, Light and temperature dependence of the carbon to chlorophyll a ratio in microalgae and cyanobacteria: implications for physiology and growth of phytoplankton, New Phytologist 106 (1987) $1-34$.

[18] M. R. Droop, Vitamin B12 and marine ecology. iv. the kinetics of uptake, growth and inhibition in Monochrysis lutheri, J. Mar. Biol. Ass. U. K. 48 (1968) 689-733.

[19] L. Rosso, J. R. Lobry, J. P. Flandrois, An unexpected correlation between cardinal temperatures of microbial growth highlighted by a new model., J Theor Biol 162 (4) (1993) 447-463.

[20] C. Piedallu, J. G. Ggout, Multiscale computation of solar radiation for predictive vegetation modelling, Ann. For. Sci. 64 (2007) 899909.

[21] Q. Bchet, A. Shilton, J. B. K. Park, R. J. Craggs, B. Guieysse, Universal temperature model for shallow algal ponds provides improved accuracy., Environ Sci Technol 45 (8) (2011) 3702-3709.

[22] T. Hirmajer, E. Balsa-Canto, J. R. Banga, DOTcvpSB, a software toolbox for dynamic optimization in systems biology., BMC Bioinformatics 10 (2009) 199.

[23] P. J. B. Williams, L. M. L. Laurens, Microalgae as biodiesel \& biomass feedstocks: Review \& analysis of the biochemistry, energetics \& economics, Energy and Environ. Sci. 3 (2010) 554-590.

[24] E. Molina Grima, E.-H. Belarbi, F. G. Acin Fernández, A. Robles Medina, Y. Chisti, Recovery of microalgal biomass and metabolites: process options and economics., Biotechnol Adv 20 (2003) 491-515. 